\title{
Urinary Sediment Dolichol Excretion in Patients with Batten Disease and Other Neurodegenerative and Storage Disorders
}

\author{
MICHAEL J. BENNETT, NIGEL J. MATHERS, FRANK W. HEMMING, IRENE ZWEIJE-HOFMAN, \\ AND GWILYM P. HOSKING \\ Departments of Chemical Pathology and The Ryegate Centre, Sheffield Children's Hospital, Sheffield, England \\ and Department of Biochemistry, University of Nottingham Medical School, Nottingham NG7 2YH and
}

Bartimeushage, Doorn, The Netherlands

\begin{abstract}
Nonesterified dolichols have been measured in the urinary sediment of 20 patients with the late infantile and juvenile forms of neuronal ceroid lipofuscinosis (Batten disease), in 15 patients with other storage and neurodegenerative disorders and in 10 control subjects. Dolichols were measured by a high performance liquid chromatographic method and were related to urinary creatinine concentration. The levels of dolichols in Batten disease were not significantly elevated when compared to the normal subjects or to patients with other neurodegenerative disorders. The highest levels seen were in two patients with mucopolysaccharidosis types II and IV, respectively. Measurement of dolichols in urinary sediment is of little value in the diagnosis of Batten disease or in furthering our understanding of the underlying primary defect. (Pediatr Res 19: 213-216, 1985)
\end{abstract}

\section{Abbreviation}

HPLC, high performance liquid chromatography

The juvenile form of neuronal ceroid lipofuscinosis is one of a group of familial severe degenerative diseases characterized histologically by an intracellular accumulation of abnormal cytosomes containing lipofuscin positive staining storage material $(6,18,19)$. There also exists infantile, late infantile, and adult forms of this disease. The basic defect is unknown and the disease is clinically marked by visual deterioration at the age of 3-7 yr, leading to blindness within a few years and by a progressive decline of mental and motor functions.

Epileptic manifestations appear and death occurs mostly between the ages of 15 and 21 yr.

At present the clinical diagnosis is confirmed by the demonstration of vacuolated lymphocytes in the peripheral blood film supplemented by examination of the urinary sediment or other tissues for the characteristic inclusion bodies peculiar to one or other of the forms-curvilinear bodies most typically in the late infantile form and finger print bodies in the case of the juvenile $(1-3,6,11)$. Rectal biopsy with subsequent histochemical staining is also employed.

The storage material has been isolated from the neurones and urinary sediment of patients with Batten disease and it has been

Received May 21, 1984; accepted October 12, 1984.

Correspondence and Reprinf Requests to M. J. Bennett, Ph.D., Department of Chemical Pathology, Sheffield Children's Hospital, Sheffield S10 2TH, England. reported that in patients with Batten disease there is greatly increased amounts of polyisoprenols of the dolichol class (C-17$\mathrm{C}-20$ ) within the storage material when compared to age-matched normals and to patients with other neurological diseases $(7,8$, $13,14)$. Furthermore it has been proposed that the measurement of dolichols in the urinary sediment could be of value in the diagnosis of Batten disease and in the screening of siblings of patients. It has also been suggested that the basic defect in the disease may be linked to dolichol metabolism (8).

In order to test the clinical usefulness of urinary sediment dolichol excretion as a noninvasive diagnostic tool in Batten disease we have measured the dolichol content of urinary sediment from 20 patients with Batten disease (juvenile neuronal ceroid lipofuscinosis) 15 patients with other storage/neurodegenerative disorders, and 10 control subjects without evidence of abnormal storage disease including four patients with the nephrotic syndrome and hence high urinary sediment output. Herein we report our observations.

\section{MATERIALS AND METHODS}

Subjects. The urines from patients with Batten disease were obtained from two sources. From nine patients with the disease in the Sheffield region we collected from one to three, 24-h urine specimens into plain bottles and frozen at $-20^{\circ} \mathrm{C}$ immediately upon receipt in the laboratory.

Early morning urine samples were obtained from 11 patients in the residential clinic "Bartimeushage," center for multiple handicapped children and young adults at Doorn in the Netherlands. These were also collected into plain bottles and immediately frozen to $-20^{\circ} \mathrm{C}$ and transported in this condition back to Sheffield. Details of individual patients are given in Table 1.

Specimens from patients previously under investigation for other storage/neurodegenerative disease were collected as random samples and immediately frozen to $-20^{\circ} \mathrm{C}$. These included three patients with nondiagnosed neurodegenerative disorders but non-Batten disease, one patient with enzymatically confirmed metachromatic leukodystrophy and 12 patients with mucopolysaccharide storage disorders the diagnosis of which had been previously confirmed by tissue enzyme analysis. These included type IH (one case), IS (one), II (two), III (five), IV (two). Nine of these specimens were kindly donated by Dr. A. Cooper, Willink Genetics Laboratory, Royal Manchester Children's Hospital, Pendlebury, Manchester, England.

Specimens were also obtained from four patients being investigated for the nephrotic syndrome as $20-\mathrm{ml}$ aliquots of vigorously mixed 24-h urine collections (the mixing was to allow a sample representative of whole urine containing sediment). Sim- 
ilarly control random urines were collected from a range of subjects including a neonate (1 month of age) and a 21 -yr-old male. The latter age approximates to the maximum survival age of patients with the juvenile form of the disease.

Equipment. The HPLC equipment consisted of a SP8770 isocratic pump (Spectra Physics Ltd., St. Albans, Herts, England) and a Kratos Spectroflow 773 spectrophotometer (Kratos Analytical Instruments, Westwood, NJ). Standard pig liver dolichols were obtained from the Sigma Chemical Company, Poole, Dorset, England.

Urine creatinines were assayed by a standard alkaline picrate method (Patchet, BP, unpublished) adapted for the Cobas Bio Centrifugal Analyser (Roche Diagnostics Ltd., Welwyn Garden City, England).

Urine dolichols were determined on samples (usually $20 \mathrm{ml}$ ) of thawed urine. The sediment in each sample was recovered by centrifugation at $2000 \times g$ for $5 \mathrm{~min}$. To each sediment was added $\left[1-{ }^{3} \mathrm{H}\right]$ dolichol $\left(10^{4} \mathrm{dpm}, 2.5 \mathrm{ng}\right)$ to act as an internal standard to enable corrections for losses during the isolation procedure. The sediment was then extracted with a mixture of chloroform and methanol $(1: 1, \mathrm{v} / \mathrm{v}, 2.5 \mathrm{ml})$ twice. The combined extracts were washed once with water $(3 \mathrm{ml})$ followed by a mixture of water and methanol $(1: 1, \mathrm{v} / \mathrm{v}, 3 \mathrm{ml})$ twice. The washed extract was taken to dryness under nitrogen and stored at $-20^{\circ}$ $C$ (usually for only a few days) until assayed by HPLC. Each fraction was dissolved in cyclohexane $(50 \mu \mathrm{l})$ and $10 \mu \mathrm{l}$ was assayed by HPLC (12) using a reversed-phase column (Ultrasphere ODS $5 \mu \mathrm{m}, 4.6 \times 250 \mathrm{~mm}$ fitted with a precolumn of Lichrosorb 5RP18, $4.6 \times 50 \mathrm{~mm}$ ) and a mixture of propan-2-ol and methanol $(7 / 3, \mathrm{v} / \mathrm{v}, 1 \mathrm{ml} / \mathrm{min})$ at $25^{\circ} \mathrm{C}$. Elution of dolichols from the column was monitored at $210 \mathrm{~nm}$ with the spectrophotometer set at 0.02 aufs. Dolichols $-17,-18,-19,-20$, and -21 were clearly resolved from each other and the amount of dolichol present was determined by measuring the peak height of dolichol -19 and comparing this with a calibration curve of peak height of dolichol -19 in standard samples of pig liver dolichols. The results are expressed in terms of total dolichol mixture taking into account the percentage composition of the mixture.

Dolichols -17 to -21 were recovered after each HPLC run and assayed for tritium content. The recovery of tritium was

Table 1. Clinical details of patients with juvenile Batten disease whose urines were analyzed in this study

\begin{tabular}{clc}
\hline Patient & Initials & $\begin{array}{c}\text { Age at time } \\
\text { of study } \\
\text { (yr) }\end{array}$ \\
\hline 1 & MS & 20 \\
2 & WM & 20 \\
3 & LT & 18 \\
4 & IB & 12 \\
5 & AR & 11 \\
6 & NH & 10 \\
7 & SB* & 9 \\
8 & JB* & 12 \\
9 & DM & 22 \\
10 & AdB $\dagger$ & 10 \\
11 & MV & 11 \\
12 & MD & 19 \\
13 & JvdB & 17 \\
14 & MN & 21 \\
15 & HS & 19 \\
16 & HdB $\dagger$ & 15 \\
17 & KK & 16 \\
18 & RvS & 21 \\
19 & JdB $\dagger$ & 12 \\
20 & PdB & 14 \\
\hline
\end{tabular}

$*,+$ Sibships. used to correct the results for losses (usually 5-15\%) during the total assay.

Dolichol levels were expressed as $\mu \mathrm{g}$ dolichol/liter of urine and as $\mu \mathrm{g} / \mathrm{mmol}$ of urinary creatinine in order to compensate for the very variable concentrations of urine obtained.

\section{RESULTS}

Figure 1 demonstrates the HPLC chromatograms for dolichol extracts in a number of the subjects studied. It is clear that the pattern of dolichols is the same in each of the samples.

Figure 2 shows the urine sediment dolichol levels in all of the subjects when expressed as $\mu \mathrm{g}$ dolichol per mmol of urinary creatinine. When expressed as $\mu \mathrm{g}$ dolichol per liter of urine a similar pattern of results was seen but with greater spread. For those patients in whom 24-h urine collections had been made, a wide range of urinary volumes were noted suggesting that many of these collections had been incomplete. Twenty-four-hour excretions were, therefore, not estimated. The age of the Batten

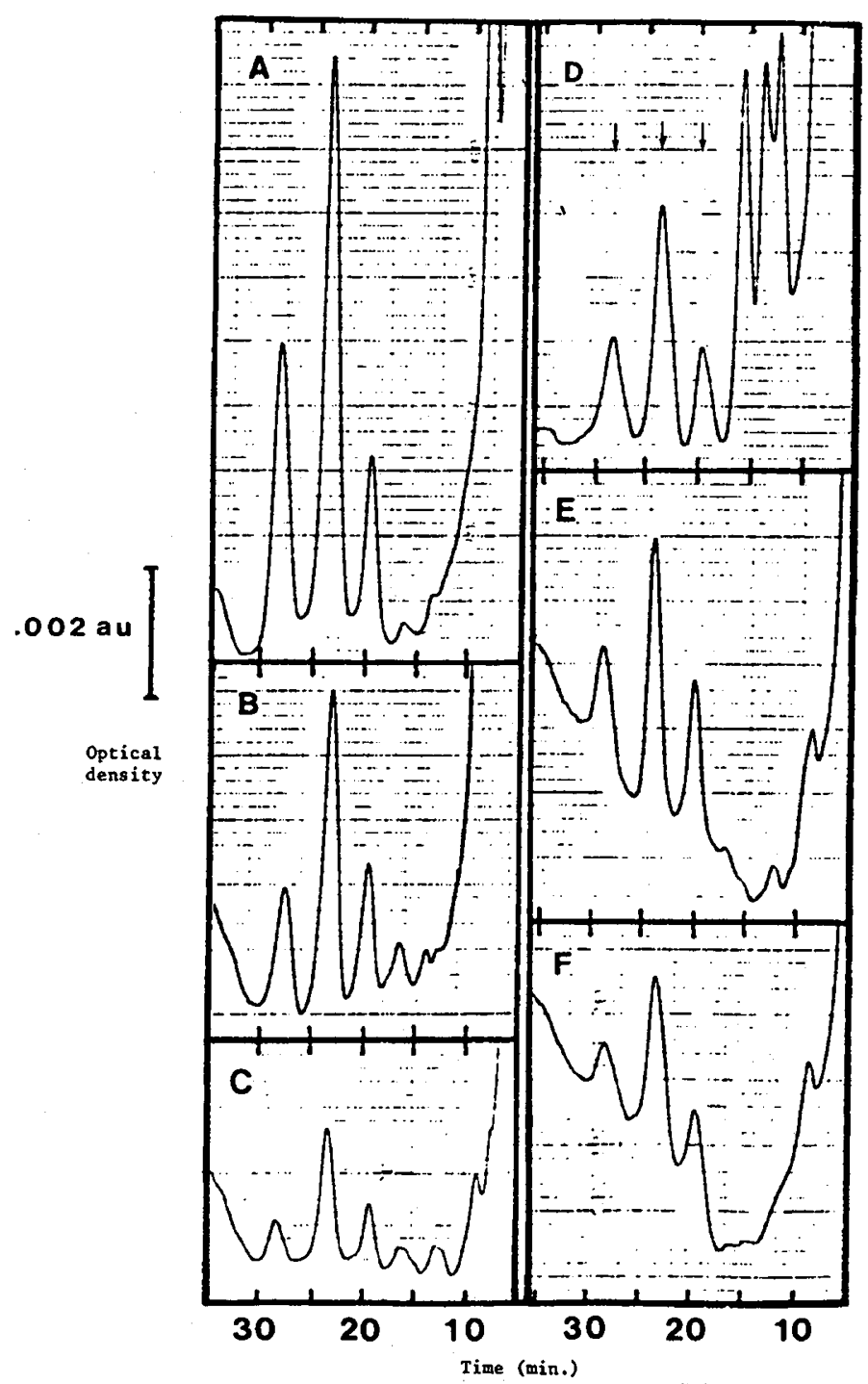

Fig. 1. Copies of HPLC traces showing the elution of dolichols from the HPLC column with time. Standard dolichols $-18,-19$, and -20 eluted routinely at $19.75,24.00$, and $28.50 \mathrm{~min}$, respectively (see arrows in panel $D$ ). Peaks observed earlier than $16 \mathrm{~min}$ are almost certainly not due to the presence of dolichols. Samples were derived from patients with the following complaints: $A$, control urine; $B$, undiagnosed neurodegenerative disorder (not Batten); $C$, metachromatic leukodystrophy; $D$, nephrotic syndrome; $E$, metachromatic leukodystrophy type II; $F$, metachromatic leukodystrophy type IV. 


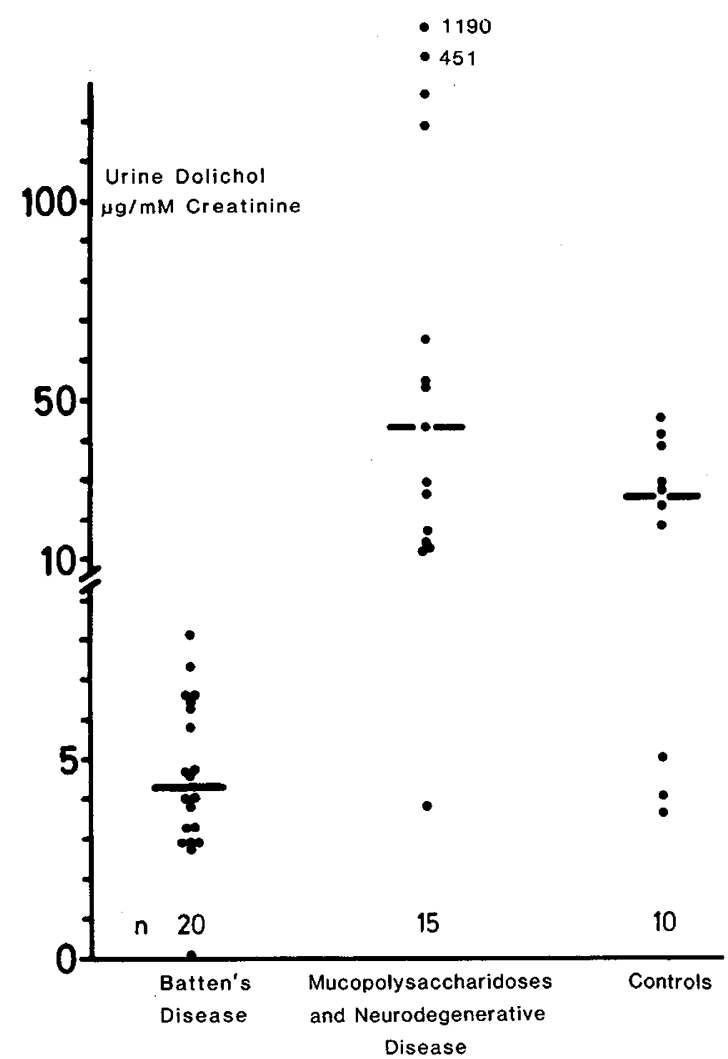

Fig. 2. Urinary sediment dolichol concentrations in the three groups of subjects studied. The results are expressed as $\mu \mathrm{g}$ dolichol $\mathrm{mmol}$ creatinine $^{-1}$. The pattern was identical when expressed as $\mu \mathrm{g}$ dolichol liter ${ }^{-1}$ but with greater spread. The mean value for the Batten disease group is significantly lower than both of the two control groups $(p<$ $0.001)$.

disease patients at the time of analysis ranged from 9-22 yr, the control group was aged from 1 month to $21 \mathrm{yr}$.

It can clearly be seen that the levels of dolichol in the control subjects and the control neurodegenerative disorder group were higher than those of the patients with Batten disease $(p<0.001$ using the Whitney-Mann Rank sum test). There was no significant difference between the two groups of control subjects.

The results obtained from one of the patients with Batten disease are of particular interest because although urine concentration was high, no nonesterified dolichol could be detected.

\section{DISCUSSION}

Dolichols are a family of polyisoprenoid alcohols with the general structure $(R=H)(4)$.

$$
\begin{aligned}
\mathrm{H}\left[\mathrm{CH}_{2}-\mathrm{C}=\right. & \left.\mathrm{CH}_{3} \mathrm{CH}-\mathrm{CH}_{2}\right]_{n} \\
\mathrm{CH}_{3} & \stackrel{\mid}{\mathrm{CH}}-\mathrm{CH}_{2}-\mathrm{CH}_{2} \mathrm{OR} \quad(\mathrm{n}=16-20)
\end{aligned}
$$

They occur in human tissues at concentrations ranging from $80-3200 \mu \mathrm{g} / \mathrm{g}$ net weight (10). The concentration increases with age of the source and there is some evidence of an association with lipofuscin. Dolichols have been isolated from the storage granules containing lipofuscin $(9,15,16)$. This view is consistent with evidence that dolichols are released from phosphodolichols ( $\mathrm{R}=$ phosphate) through the action of a phosphatase in the lysosomes (17).

Phosphodolichol is the functional form of the compound acting as an essential and rate-limiting coenzyme in the $\mathrm{N}$-glycosylation of proteins. There are several reports of interfer- ence in this process resulting in the failure to produce functional glycoproteins, e.g. the acetylcholine receptor (reviewed in Reference 5). These observations have stimulated an interest in the relationship between concentrations of dolichol and phosphodolichol in human tissues especially in neurodegenerative disorders. Elevated concentrations of dolichols in the brain associated with Alzheimer's disease and with Batten disease have been reported (16). It appears that changes in the concentration of dolichol are not restricted to neural tissue and these same authors describe the presence of increased concentrations of dolichol in epithelial cells of the kidney in all forms of neuronal ceroid lipofuscinosis.

Figure 2 clearly demonstrates that there is a wide variation in the amount of dolichol associated with sediment of urine in different individuals. It is evident that comparisons based on dolichol per unit volume of urine or per unit weight of creatinine in the urine do not allow specific diagnosis of Batten disease. $\mathrm{Ng}$ Ying and Wolfe (8) assayed the sediment from much larger volumes of urine and related the dolichol-content to the total lipid of the sediment. Reporting on samples from a different range of medical conditions from those assayed herein they observed that a high ratio of dolichol to total lipid of urinary sediment was characteristic of Batten disease. The smaller volumes of urine and hence smaller quantities of sediment available in the experiments reported herein did not allow an accurate lipid-assay of the sediment. However, it is unlikely that a dolichol assay per unit of cellular sediment would have materially altered our conclusions. For the dolichol content of the cellular sediment in the Batten patients to be routinely and significantly higher than in those described in the non-Batten groups would require the latter samples to have a cellular content many fold greater than the Batten samples. This greater cellularity would not be expected. Wolfe et al. (16) reported similar results for their data when expressed per $100 \mathrm{ml}$ urine or per gram creatinine.

There is one clear incidence of a case of Batten disease which does not show detectable levels of dolichol at all (Fig. 2) and this would clearly be a false-negative if urinary dolichol levels were to be used as a screening procedure.

Our studies suggest, therefore, that the specific basic lesion in Batten disease is not directed toward abnormalities in dolichol metablism and that current diagnostic methods remain the best that are available to us.

Acknowledgment. The authors would like to acknowledge the valuable technical assistance of Mr. M. Smyley in the HPLC assays.

\section{REFERENCES}

1. Carpenter S, Karpati G, Andermann F, Jacob JC, Anderman E 1977 The ultrastructural characteristics of the abnormal cytosomes in Batten-Kufs disease. Brain 100:137

2. De Baecque C 1975 Diagnosis of neuronal ceroid-lipofuscinosis by electron microscopy of urinary sediment. N Engl J Med 292:1408

3. De Baecque C, Pollack AM, Suzuki K 1976 Systemic pathology in late infantile storage disease with curvilinear bodies. Arch Pathol Lab Med 100:139

4. Hemming FW 1974 Lipids in glycan biosynthesis in MTP. In: Goodwin TW (ed) International Reviews of Science, Biochemistry Series 1, Vol 4. Butterworths, London, pp 39-57

5. Hemming FW 1982 Control and manipulation of the phosphodolichol pathway of protein N-glycosylation. Biosci Rept 2:203

6. Lake BD 1981 Metabolic disorders: general considerations. In: Berry CL (ed) Paediatric Pathology. Springer-Verlag, Berlin, pp 617-635

7. Ng Ying Kin, NMK, Palo J, Haltia M, Wolfe LS 1983 High levels of brain dolichols in neuronal ceroid-lipofuscinosis and Senescence. J Neurochem 40:1465

8. Ng Ying Kin NMK, Wolfe LS 1982 Presence of abnormal amounts of dolichols in the urinary sediment of Batten disease patients. Pediatr Res 16:530

9. Pullarkat RK, Reha H 1982 Accumulation of dolichols in the brains of the elderly. J Biol Chem 257:5991

10. Rupar CA, Carroll KK 1978 Occurence of dolichol in human tissues. Lipids 13:291

11. Schuurmans Stekhoven JH, van Haelst UJGM, Joosten EMG, Loonen MCB 1977 Ultrastructural study of the vacuoles in the peripheral lymphocytes in juvenile amaurotic idiocy. Acta Neuropathol 38:137

12. Tavares IA, Coolbear T, Hemming FW 1981 Increased hepatic dolichol and dolichol and dolichol phosphate-mediated glycosylation in rats fed choles- 
terol. Arch Biochem Biophys 207:427

13. Wolfe LS, Ng Ying Kin NMK, Baker RR 1981 Batten disease and related disorders: new findings on the chemistry of the storage material. In: Callahan JW, Lowden JA (eds) Lysosomes and Lysosomal Storage Diseases. Raven Press, New York, pp 315-330

14. Wolfe LS, Ng Ying Kin NMK, Baker RR, Carpenter S, Andermann F 1977 Identification of Retinoyl complexes as the autofluorescent component of the neuronal storage material in Batten disease. Science 195:1360

15. Wolfe LS, Ng Ying Kin NMK, Palo J, Haltia M 1982 Raised levels of cerebral cortex dolichols in Alzheimers disease. Lancet 1:99
16. Wolfe LS, Ng Ying Kin NMK, Palo J, Haltia M 1983 Dolichols in brain and urinary sediment in neuronal ceroid lipofuscinosis. Neurology 33:103

17. Wong TK, Lennarz WJ 1982 The site of biosynthesis and intracellular deposition of dolichol in rat liver. J Biol Chem 257:6619

18. Zeman W 1974 Studies in the neuronal ceroid-lipofuscinosis. J Neuropathol Exp Neurol 33:1

19. Zeman W, Donahue S, Dyken P, Green J 1970 The neuronal ceroid-lipofuscinosis (Batten-Vogt syndrome). In: Vinken PJ, Bruyn GW (eds) Handbook of Clinical Neurology, Vol 10. North Holland, Amsterdam, pp 588-679

\title{
N-Acetylneuramin Lactose Sulfate: A Newly Identified Nutrient in Milk
}

\author{
JOHN A. STURMAN, YONG Y. LIN, TETSUO HIGUCHI, AND J. H. FELLMAN
}

Departments of Pathological Biochemistry and of Human Development and Nutrition, Institute for Basic Research in Developmental Disabilities, Staten Island, New York 10314 [J.A.S., Y.Y.L.]; JEOL (U.S.A.), Inc., Cranford, New Jersey 07016 (T.H.); and School of Medicine, Department of Biochemistry, Oregon Health Sciences University, Portland, Oregon 97201 [J.H.F.]

\begin{abstract}
The identity of a sulfate ester in rat milk has been determined to be $\mathrm{N}$-acetylneuramin lactose sulfate. This sulfate ester is present in rat mammary tissue and in human milk. The presence of this compound offers an explanation for the simultaneous delivery of sulfate and calcium via the milk, two essential nutrients in early life, without precipitation of calcium sulfate in the milk. $\mathbf{N}$ acetylneuramin lactose sulfate is hydrolyzed in the gut of the neonate and absorbed as inorganic sulfate. This is the first report suggesting that this ester may be of nutritional importance. (Pediatr Res 19: 216-219, 1985)
\end{abstract}

The delivery of nutrients to dependent offspring via the milk is an important process for normal development, although many of the constituents remain unidentified at present. Even in man, where the greatest research effort has been expended, many nutrients and growth factors have yet to be identified. This situation is exemplified by the different metabolic responses in human infants fed human milk or a variety of synthetic formulas based on partially purified proteins (1-8). In general the concentration of most amino acids in plasma and urine is greater in infants fed formulas than in those fed human milk, although taurine is a notable exception. The role of taurine in the nervous system, especially during development, has received much attention during the last decade, especially since the discovery that cats and kittens fed synthetic diets containing partially purified proteins suffered from retinal and tapetal degeneration (9-18).

Received March 23, 1984; accepted October 16, 1984

Correspondence to Dr. John A. Sturman, Developmental Neurochemistry Laboratory, Department of Pathological Biochemistry, Institute for Developmental Disabilities, 1050 Forest Hill Road, Staten Island, NY 10314.

Supported by the Office of Mental Retardation and Developmental Disabilities of the State of New York.
More recently it has been shown that rhesus monkeys raised on a synthetic human infant formula have damaged cone photoreceptors (19). Radioactive taurine injected intraperitoneally into lactating female rats is secreted into the milk, and allows estimation of the contribution of milk taurine to the brain and other tissues $(20,21)$. During these investigations milk was found to contain a radioactive sulfur-containing compound which was eluted from an ion exchange column in the proximity of the solvent front (Fig. 1). Most tissues and fluids contained only radioactive taurine, but in a few, such as plasma, urine, and feces, radioactive inorganic sulfate was also detected. A radioactive compound found in milk was distinguished from inorganic sulfate by the fact that a negligible portion was precipitated by barium chloride. Small amounts of the compound, along with inorganic sulfate, were detected in the viscera of the suckling pups. The identification of this compound as $\mathrm{N}$-acetylneuramin lactose sulfate and a discussion of its potential nutritional role is the subject of this communication.

\section{MATERIALS AND METHODS}

Lactating rats were injected intraperitoneally with $\left[{ }^{35} \mathrm{~S}\right]$ taurine (specific activity $51 \mathrm{mCi} / \mathrm{mmol}$ ) or $\left[{ }^{35} \mathrm{~S}\right.$ ]inorganic sulfate (specific activity $78 \mathrm{mCi} / \mathrm{mmol}$ ). Both radiochemicals were purchased from Amersham. The experiments with $\left[{ }^{35} \mathrm{~S}\right]$ taurine have been previously described in detail (22). The experiments with $\left[{ }^{35} \mathrm{~S}\right]$ inorganic sulfate were performed as follows: Four lactating dams were injected with $500 \mu \mathrm{Ci}\left[{ }^{35}\right.$ S] inorganic sulfate on days 3 and 4 after birth, and milk samples obtained on days 4,5 , and 6 (on day 4 the sample was obtained $24 \mathrm{~h}$ after the first injection, and prior to the second injection; on day 5 the sample was obtained $24 \mathrm{~h}$ after the second injection). After the third milking, mother and pups were killed and liver, brain, and mammary tissue dissected from the mother, and liver, brain, stomach milk, and carcass retained from the pups for later analysis. Milk samples were obtained after separating the pups from the mother for $4 \mathrm{~h}$, 